\title{
Balkanologie
}

Balkanologie Revue d'études pluridisciplinaires

Vol. VI, $n^{\circ}$ 1-2 | 2002

Volume VI Numéro 1-2

\section{La péninsule de Prevlaka}

The Prevlaka Peninsula

\section{Diane Masson}

\section{OpenEdition}

Journals

Édition électronique

URL : http://journals.openedition.org/balkanologie/443

DOI : 10.4000/balkanologie.443

ISSN : 1965-0582

\section{Éditeur}

Association française d'études sur les Balkans (Afebalk)

\section{Édition imprimée}

Date de publication : 1 décembre 2002

Pagination : 61-68

ISSN : 1279-7952

\section{Référence électronique}

Diane Masson, «La péninsule de Prevlaka », Balkanologie [En ligne], Vol. VI, n 1-2 | 2002, mis en ligne le 03 février 2009, consulté le 17 décembre 2020. URL : http://journals.openedition.org/balkanologie/443 ; DOI : https://doi.org/10.4000/balkanologie.443

(c) Tous droits réservés 


\title{
LA PÉNINSULE DE PREVLAKA
}

\author{
Diane Masson*
}

Au printemps 2002, à la suite de la visite du ministre croate des Affaires étrangères Tonino Picula à Belgrade, la question de Prevlaka semblait être en voie de règlement, dix ans environ après le début de ce contentieux micro-territorial entre la Croatie et la Yougoslavie. Bien que petite par sa taille, deux kilomètres de long et 500 mètres de large, la péninsule de Prevlaka occupe une place stratégique. Située à l'extrémité méridionale du littoral croate et à deux kilomètres seulement de la frontière monténégrine, Prevlaka, base militaire à l'époque communiste, contrôle l'entrée des Bouches de Kotor (Boka kotorska). Le conflit de Prevlaka a une place à part dans les guerres yougoslaves : il est en effet resté entièrement bilatéral. Ainsi, malgré la présence de troupes de l'ONU, la communauté internationale n'est pas intervenue pour trouver une solution politique, le règlement du contentieux se trouvant dans le camp des gouvernements croate et yougoslave.

\section{APERÇU HISTORIQUE}

La république de Croatie présente une curieuse discontinuité territoriale, car sa portion la plus méridionale n'est pas directement rattachée au territoire national. Elle en est séparée par une bande de 21,2 kilomètres qui relève de la Bosnie-Herzégovine (incluant le petit port de Neum). L'histoire explique cette anomalie. Le territoire croate isolé par le corridor de Neum correspond en effet exactement à celui de l'ancienne république de Raguse (Dubrovnik), qui fut un petit Etat souverain, quoique tributaire de l'Empire ottoman, jusqu'en 1808 .

\footnotetext{
• Docteur en science politique de l'Institut d'Etudes Politiques de Paris.
} 
C'est dans le premier quart du XV siècle que les Ragusains établirent leur contrôle sur la petite plaine des Konavli et sur les petits reliefs qui la prolongent au sud, jusqu'à l'étroite pointe de Prevlaka. Ce n'est pas un hasard si l'ultime hameau ragusain, sur les Bouches de Kotor, se nomme Konfin. Venise, grande rivale commerciale de Dubrovnik, détenait la Dalmatie jusqu'à l'embouchure de la Neretva ainsi que le pourtour des Bouches de Kotor (que les sources de l'époque appelaient l'Albanie vénitienne). Menacés d'encerclement, les Ragusains obtinrent par le traité de Karlowitz (Sremski Karlovci) en 1699 que deux bandes de territoire ottoman fissent tampon entre leur territoire et les possessions vénitiennes : la bande de Neum au nord-ouest, celle de Sutorina au sud-est. La première, comme nous l'avons vu, reste inscrite dans le découpage politique contemporain. La seconde, qui offrait à la BosnieHerzégovine une lucarne peu commode sur les Bouches de Kotor, fut supprimée en 1945, lors du départage des républiques fédérales de Yougoslavie.

Ces frontières, fixées en 1699 et confirmées en $\mathbf{1 7 1 8}$, furent maintenues en 1815 , lorsque l'Autriche devint le propriétaire unique des trois portions de littoral disjointes. L'usage s'imposa au $\mathrm{XIX}^{\mathrm{e}}$ siècle d'englober l'ex-territoire ragusain et l'ex-Albanie vénitienne sous le vocable de Dalmatie. Les frontières de Neum et de Sutorina restèrent internationales, de facto jusqu'en 1878 , de jure jusqu'en 1908 (avec l'annexion de la Bosnie-Herzégovine). On les retrouve, sous la forme de divisions administratives, dans le srez de Dubrovnik, que le royaume de Yougoslavie rattacha d'abord à la banovina de la Zeta, puis, en août 1939, à la grande banovina croate. La persistance de tracés frontaliers parfois très anciens est une caractéristique de la partie occidentale des Balkans, où elle fut perpétuée par le pouvoir habsbourgeois. C'est une des composantes du droit historique qui occupe une place majeure dans les argumentaires nationaux hongrois et croate. Ce type d'argument n'a pourtant qu'une force de persuasion très médiocre dans les autres parties des Balkans, où l'approche territoriale est beaucoup plus pragmatique.

\section{LES ORIGINES DU CONTENTIEUX}

Au sein de la Yougoslavie titiste, Prevlaka, cette étroite bande de terre qui s'avance dans l'Adriatique, appartenait à une zone militarisée, siège d'une base navale, située à l'intérieur des frontières administratives de la république socialiste fédérative de Croatie. De violents combats s'y engagèrent entre l'Armée populaire yougoslave (JNA) et les troupes croates à la suite de la déclaration d'indépendance de la Croatie le 25 juin 1991. Cette dernière revendiquait la terre, sur laquelle elle avait décidé d'exercer sa souveraineté, alors que la Yougoslavie affirmait de son côté être propriétaire de la base navale qui s'y 


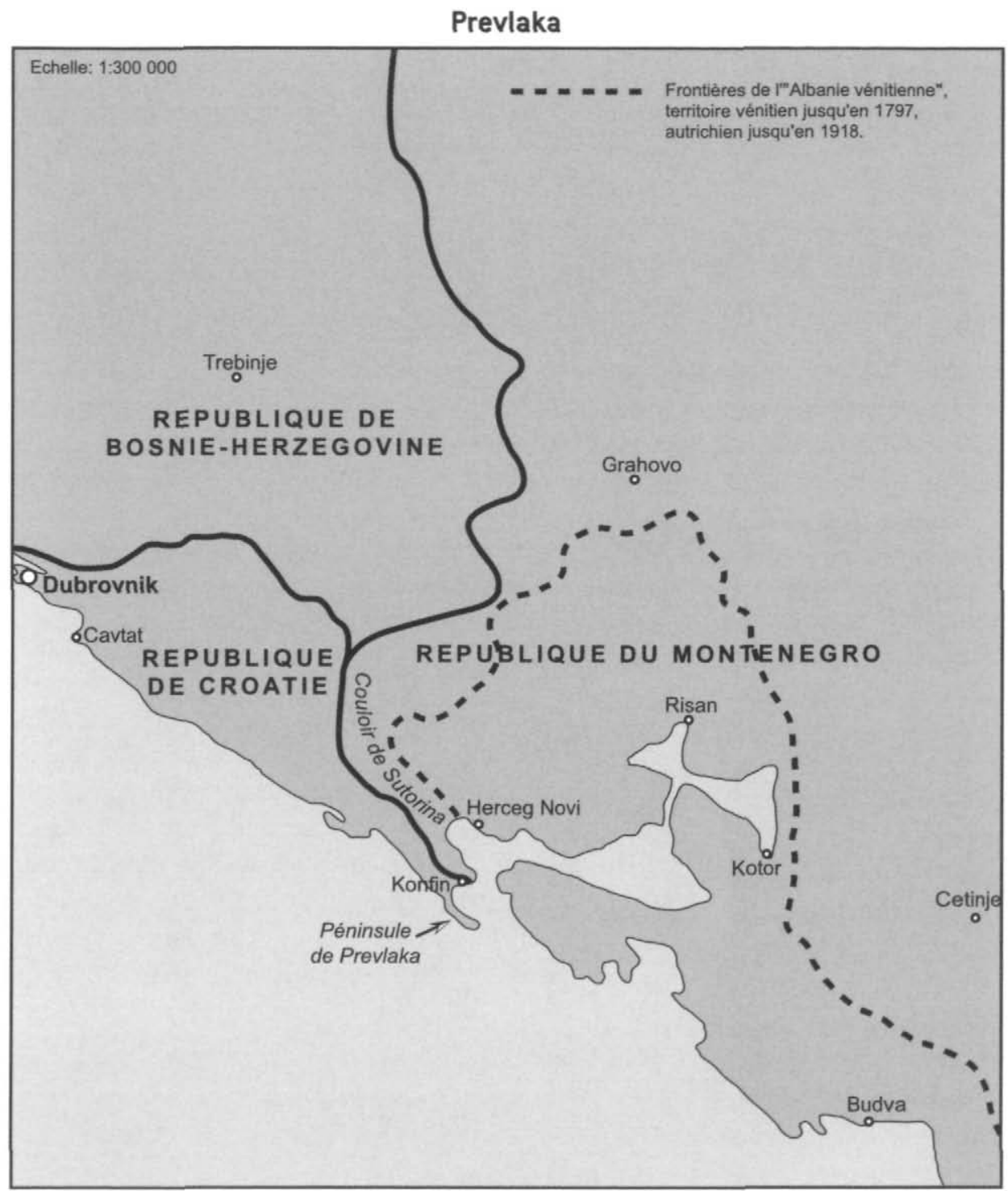

trouvait. La JNA échoua dans sa tentative de reprise de la péninsule et un cessez-le-feu mit fin en février 1992 aux opérations militaires à la faveur d'une intervention de l'ONU. Le 30 septembre 1992, une déclaration commune était somme toute signée à Genève par les présidents croate et yougoslave, concernant la démilitarisation de la péninsule ainsi placée sous contrôle de l'ONU' ${ }^{1}$.

${ }^{1}$ Allcock (John), Milivojevic (Marko), Horton (John J.), eds. Conflict in the Former Yugoslavia. An Encyclopedia. Denver/Santa Barbara/Oxford : ABC-Clio, 1998, pp. 225-226. 
En adoptant la Résolution 740 (1992) et le plan de maintien de la paix en Croatie, qui appelait " toutes les forces de la JNA déployées en Croatie à quitter cette république ", le Conseil de Sécurité de l'ONU confirmait l'appartenance de Prevlaka aux frontières internationalement reconnues de la $\mathrm{Croatie}^{2}$. En quittant les lieux, les militaires yougoslaves confiaient la base aux Nations Unies, partant du principe qu'elles en seraient temporairement le gardien jusqu'à ce que la Croatie et la Yougoslavie aient réglé leurs problèmes liés à la propriété territoriale et à la sécurité des Bouches de Kotor ${ }^{3}$. Pourtant, la solution à ce micro-conflit était loin d'être trouvée, des résolutions de l'ONU prolongeant depuis cette date le mandat des casques bleus de six mois en six mois.

\section{MISSION OU DÉMISSION ?}

Pendant les guerres de 1991-1995, la Croatie et la Yougoslavie n'ont pas progressé dans le règlement de la question de Prevlaka. Elle fut aussi l'une des absentes des accords de paix de Dayton. A leur retour des Etats-Unis en novembre 1995, le président croate Franjo Tudjman et son ministre des Affaires étrangères Mate Granić devaient expliquer cet état de fait à la population : le premier affirma qu'une éventuelle cession de Prevlaka serait uniquement acceptable si elle répondait aux intérêts de la Croatie ; le second annonça pour sa part que le sujet n'avait pas été réglé à Dayton, car il serait à l'ordre du jour après la reconnaissance mutuelle de la Yougoslavie et de la Croatie dans leurs frontières actuelles, comme cela était prévu dans les accords de paix 4 . Au cours des pourparlers de Dayton, la délégation yougoslave aurait à plusieurs reprises proposé à la Croatie d'échanger Prevlaka contre un autre territoire, offre qui fut déclinée ${ }^{5}$. Par la suite, Zagreb n'aurait pas indiqué de positionnement officiel car le président Tudjman espérait tout de même qu'un échange de Prevlaka contre un territoire d'Herzégovine, situé derrière Dubrovnik, serait possible ${ }^{6}$. Quoi qu'il en soit, la Croatie et la Yougoslavie signaient en août 1996 un accord

\footnotetext{
2 "Croatian Prevlaka, A legal perspective ". Croatian Mission to the United Nations. http://www.un.int/croatia/prevlaka.html.

${ }^{3}$ Calver (Richard). " Peacekeeping that lives up to its name n. United Nations peace operations in 2001. The Year in Review. http://www.un.org.

4 Vjesnik, 22 novembre 1995.

5 “ Croatian Prevlaka, A legal perspective ", (art. cit.).

6 Thompson (Mark). "Croatie : Arrivée de troupes dans Prevlaka ", Institute for War \& Peace Reporting, 23 avril 1999.
} 
de normalisation de leurs relations, dont l'article 4 était consacré au contentieux de Prevlaka :

Les parties contractantes s'accordent à résoudre la question contestée de Prevlaka par le biais de négociations, afin d'éviter la présence de tensions des deux côtés dans la région, qui devraient contribuer à la totale sécurité de la partie appartenant à la république de Croatie dans la région de Dubrovnik et de la partie appartenant à la république fédérale de Yougoslavie dans les Bouches de Kotor. Les parties contractantes devront résoudre cette importante question à travers des négociations conformes à l'esprit des Nations Unies et dans de bonnes relations de voisinage?.

Il faudra cependant attendre jusqu'à l'été 1998 pour que la situation de Prevlaka soit directement abordée par les deux pays concernés. Un projet d'accord croate fut officiellement soumis à la RFY le 15 juin 1998, stipulant :1) la délimitation des frontières internationales existantes ; 2) l'ouverture permanente des postes frontières de Debeli Brijeg et de Konfin ; 3) la délimitation des frontières maritimes ; 4) la démilitarisation asymétrique selon la proportion $1: 2,5$ en faveur de la partie yougoslave pour une période de cinq ans ; 5) la vérification de l'application de ces accords par une commission inter-étatique mixte ${ }^{8}$. Le 10 juillet 1998, la réponse, ou proposition yougoslave, prônait quant à elle les points suivants : 1) la délimitation des frontières terrestres et maritimes ; 2) la démilitarisation permanente de Prevlaka ; 3) l'établissement d'un poste frontière permanent le long de l'autoroute Herceg Novi-Sutorina-Dubrovnik ; une régulation de la circulation effectuée conjointement par les municipalités yougoslaves et croates ${ }^{9}$. Pour les autorités de Belgrade, point de discorde majeur, Prevlaka représentait un problème territorial qui pourrait uniquement se résoudre par la modification des frontières internationales, solution - nonconforme aux accords de Dayton - inacceptable pour la Croatie et pour la communauté internationale. Ainsi, malgré la nomination d'une commission commune sur Prevlaka et la tenue de quatre réunions entre septembre 1998 et mars 1999, les négociations ont continué d'achopper, avant d'être bloquées pendant les bombardements de l'OTAN sur la RFY. Il apparaît clairement que Slobodan Milošević a mis en œuvre diverses tactiques dans le but de reporter toute négociation sérieuse, ce qui constituait pour lui un moyen de pression contre la Croatie, mais aussi contre le Monténégro, qui avait boycotté les négociations de la commission commune dès octobre 1998, pour cause de désaccord avec le régime de Belgrade ${ }^{10}$.

\footnotetext{
${ }^{7}$ In : " Croatian Prevlaka, A legal perspective ", (art. cit.).

${ }^{8}$ Ibid.

9 Ibid.

${ }^{10}$ Ibid. L'arrivée de Milo Djukanović (hostile au pouvoir de S. Milošević) à la tête du Monténégro en 1998 a en effet changé les relations serbo-monténégrines et facilité les relations croato-monténégrines.
} 
Créée par la résolution 1038 du Conseil de sécurité le 15 janvier 1996, la Mission d'Observation des Nations Unies à Prevlaka (MONUP) remplaça l'ONURC. Composée de vingt-sept observateurs militaires, ses objectifs étaient de " contrôler la démilitarisation de la péninsule dans le but de contribuer à résoudre la tension et les divergences entre la république de Croatie et la RFY " ${ }^{11}$. Pourtant, selon le journaliste anglais Mark Thompson, qui fut également l'un des membres de la mission des Nations Unies à Prevlaka en 1997, aucune des parties n'aurait respecté la zone démilitarisée : les troupes yougoslaves ne se sont jamais retirées de leurs positions près des frontières bosniaques, et la "police spéciale" croate s'est installée dans des "bunkers" aux environs de Prevlaka $^{12}$. Malgré tout, la région est globalement restée calme depuis 1995, excepté en avril 1999, lorsque des soldats yougoslaves furent sur le point de prendre le contrôle du poste frontière de la péninsule, mais ce sans conséquences notables ${ }^{13}$.

\section{L'ALTERNANCE DU POUVOIR À ZAGREB ET À BELGRADE}

Les transformations politiques de l'année 2000 en Croatie et en Serbie marquaient indéniablement le début d'une nouvelle stabilité régionale pour les Balkans. Allaient-elles pour autant sonner le glas du micro-conflit de Prevlaka ? Le 4 novembre 2000 , Zoran Djindjić, l'un des principaux représentants de l'Opposition démocratique de Serbie (DOS) et futur Premier ministre serbe, affirmait à la télévision croate que " la péninsule de Prevlaka devrait appartenir au Monténégro " et que "ce serait un geste de bonne volonté de la Croatie de l'accepter ${ }^{14}$. Cette déclaration a bien entendu suscité de vives réactions auprès des dirigeants croates, qui attendaient au contraire une véritable rupture avec la politique de Slobodan Milošević, remettant en cause l'appartenance territoriale et géographique de Prevlaka à la Croatie. Malgré ces débuts quelque peu incertains, le ministre yougoslave des Affaires étrangères Goran Svilanović exprima assez rapidement la volonté de son pays de résoudre le différend sur la péninsule de Prevlaka avec la Croatie ${ }^{15}$. Sa première visite officielle en Croatie en décembre 2001 prouvait effectivement le réchauffement des relations entre les deux pays. A cette occasion, Tonino Picula énonça les dix

\footnotetext{
${ }^{11} \mathrm{http}$ ///www.un.org.

${ }^{12}$ Thompson (Mark), (art. cit.).

${ }^{13}$ Ibid.

14 Vjesnik, 5 novembre 2000.

15 Danas, 26 mai 2001.
} 
points constituant une entrave à la normalisation entre Zagreb et Belgrade, parmi lesquels figurait la question de Prevlaka, " qui devait être réglée dans les prochains mois avec l'aide d'une commission mixte ". Cette dernière fut formellement mise en place lors de la même visite de son homologue serbe ${ }^{16}$. Dans une lettre adressée au Conseil de Sécurité, l'ambassadeur croate aux Nations Unies Ivan Simonović demandait pour sa part que le mandat de la MONUP " soit prolongé pour une dernière période de six mois et prenne fin le 15 juillet $2002 n^{17}$. Il en appelait également à l'aide du Conseil, afin qu'il insiste pour que la commission croato-yougoslave, créée pour délimiter le tracé de la frontière terrestre et maritime dans cette région, achève dans les meilleurs délais ses travaux ${ }^{18}$. Le retrait de la MONUP en juillet 2002 était également approuvé par les autorités de Belgrade.

Du point de vue des frontières, les deux ministres des Affaires étrangères se sont mis d'accord à Zagreb le 10 avril 2002 sur le principe d'une reconnaissance des anciennes frontières entre la Croatie et le Monténégro, condition qui permettrait la complète normalisation de la situation dans la région de Prevlaka, une démilitarisation effective, ainsi que le retrait des observateurs de $l^{\prime} \mathrm{ONU}^{19}$. Deux semaines plus tard, un protocole sur les frontières, le premier depuis l'éclatement du conflit en 1991, a été signé à Belgrade par Tonino Picula et Goran Svilanović ${ }^{20}$. D'après leur déclaration commune, le règlement définitif du statut de la péninsule de Prevlaka devrait avoir lieu avant la fin de l'année $2002^{21}$.

Tant que les gouvernements croate et yougoslave n'avaient pu s'entendre sur les mesures nécessaires pour déterminer qui était propriétaire de la péninsule de Prevlaka et qui était en charge de la sécurité des Bouches de Kotor, les Nations Unies étaient demeurées sur place, prolongeant le mandat de leurs observateurs tous les six mois à partir d'octobre 1992, mais sans essayer d'intervenir pour aider les parties en présence à trouver une solution politique. Il existait toutefois une différence majeure dans les objectifs des deux républiques : pour le Monténégro, il s'agissait de construire son territoire, c'est-àdire d'occuper le littoral et de descendre vers l'Adriatique, volonté qui s'est affirmée par phases. La Croatie avait quant à elle une position défensive de protection de ses anciens acquis.

\footnotetext{
${ }^{16}$ Vjesnik, 14 décembre 2001.

${ }^{17}$ Vjesnik, 9 janvier 2002.

${ }^{18}$ Ibid.

${ }^{19}$ RFL/RL Research Report, 11 avril 2002.

${ }^{20}$ Danas, 24 avril 2002.

${ }^{21}$ Ibid.
} 
La donne se compliqua davantage à partir de 1998 lorsque les pourparlers devinrent triangulaires, intégrant un Monténégro désormais opposé au diktat de Belgrade. Si la responsabilité principale de l'achoppement des négociations a le plus souvent été rejetée sur le président Milošević, force est de constater que la position de son homologue croate Franjo Tudjman n'a pas toujours été des plus cristallines. Les recompositions politiques en Serbie et en Croatie allaient permettre un nouveau départ. A l'automne 2002, l'avenir de Prevlaka ne constitue plus une menace pour la paix et la sécurité de la Croatie et de la Serbie-Monténégro. Bien que le mandat de la MONUP ait finalement été prolongé pour une durée de six mois en juillet 2002, le régime transitoire de contrôle des frontières semble toucher à sa fin, à l'heure où les gouvernements concernès n'estiment plus nécessaire la présence d'une mission de maintien de la paix le long de leurs frontières. 